HA as a non-invasive marker for prognosis in patients with chronic liver disease of varying aetiology.

\section{P07 SOCIO-DEMOGRAPHIC AND BEHAVIOURAL DETERMINANTS OF ABNORMAL LIVER FUNCTION IN A MULTI ETHNIC POPULATION IN THE UK: THE LOLIPOP STUDY}

\section{doi:10.1136/gutjnl-2011-300857a.7}

${ }^{1} S$ D S Fraser, ${ }^{1} \mathrm{~J}$ Parkes, ${ }^{1} \mathrm{P}$ J Roderick, ${ }^{2} \mathrm{~J} S \mathrm{~S}$ Kooner, ${ }^{2} \mathrm{~J}$ Chambers. ${ }^{1}$ University of Southampton, UK; ${ }^{2}$ Imperial College, London, UK

Introduction Fatty liver disease (FLD) is common, with an estimated prevalence between 10 and $24 \%$ of the general population. Most patients are asymptomatic, and identified via incidental abnormal liver function tests, particularly alanine transaminase (ALT) and $\gamma$-glutamyltransferase (GGT). There are few population data on the prevalence or determinants of abnormal liver function.

Aim To determine the characteristics and associations of people with elevated values 1.5 times normal of both ALT and GGT in a population sample (upper limit of normal (ULN)=ALT $\$ 37$ \$31 IU/ 1; GGT $\$ 55$ \$38 IU/1) as an indicator of FLD.

Method The London Life Sciences Prospective Population Study (LOLIPOP study) is a population based cohort study of cardiovascular risk and outcomes in west London, an area with a high ethnic minority population. Adults aged 35-74 from 58 general practices were invited to cardiovascular screening which included questionnaire, clinical measurement and blood sampling. Response rate was $62 \%$. Cross sectional data were obtained on 31507 , European white $(n=9222)$, Indian Asian $(n=19769)$ and Black $(n=2516)$. ALT and GGTwere measured from a single serum sample. To identify key socio-demographic variables and potentially modifiable lifestyle factors, including BMI and alcohol intake, we used logistic regression models examining associations with elevated ALT and GGT.

Results The number with GGT and ALT measures was 31465 (99.9\% of total). The prevalence of elevated GGT above 1.5 times the ULN (GGT 1.5) was 10.4\% (3265/31 465), for ALT 8\% (2517/31 465) and for both $3.2 \%$ (996/31465). $40 \%$ of those with ALT1.5 had GGT1.5, and $32 \%$ of those with GGT 1.5 had ALT 1.5. The independent odds of having both raised were increased in: younger people (OR 3.00 (95\% CI 2.28 to 3.94) for 35-44 compared to 65-74 age group), south Asians (OR 1.29 (95\% CI 1.10 to 1.51 ) compared to White), greater alcohol intake (OR 6.52 (95\% CI 5.36 to $7.95)$ for $>28$ units per week compared to no alcohol), raised BMI (OR 2.34 (95\% CI 1.94 to 2.83 ) obese compared to normal), and diabetes (OR 1.32 (95\% CI 1.07 to 1.63), with a dose related gradient for alcohol consumption. Gender, deprivation, and taking statin were not significantly associated, and no interaction observed between alcohol and gender or BMI.

Conclusion The prevalence of abnormal liver function tests, suggesting FLD, was common (directly standardised prevalence of $3.3 \%$ in this age group in the England and Wales population). BMI, and alcohol independently increased risk across the range of their values, highlighting the importance of considering both in preventing severe liver disease and counselling patients.

\section{P08 SPONTANEOUS BACTERIAL PERITONITIS PROPHYLAXIS: REDUCING THE INCIDENCE OF C DIFFICILE INFECTION}

\section{doi:10.1136/gutjnl-2011-300857a.8}

${ }^{1} \mathrm{H}$ Jafferbhoy, ${ }^{1} \mathrm{M}$ H Miller, ${ }^{1} \mathrm{C}$ Shekar, ${ }^{2} \mathrm{M}$ Lockhart, ${ }^{1} \mathrm{~J}$ F Dillon. ${ }^{1}$ GUT Group Biomedical Research Institute, Dundee University; ${ }^{2}$ Department of Microbiology, Ninewells Hospital

Introduction Spontaneous bacterial peritonitis is a serious and life-threatening complication of cirrhosis, especially common in hospitalised patients. Antibiotic prophylaxis is effective but can lead to an increased incidence of hospital-acquired infections such as Clostridium difficle.

Aim We evaluated whether two alternative prophylaxis agents were equally efficacious in preventing SBP, and the impact on risk of $C$ difficile infection.

Method A consecutive, cohort study of hospitalised patients with cirrhosis and ascites, over a 3-year period in a tertiary hospital. In the first cohort (2007-2009), ascitic patients requiring prophylaxis received Norfloxacin $400 \mathrm{mg} / \mathrm{d}$ during their hospital admission. In the second cohort (2009-2010) patients received prophylactic Co-trimoxazole $960 \mathrm{mg} / \mathrm{d}$ during their hospital admission. Data were extracted by case note review and the two cohorts compared. Results 174 patients admitted during 2007-2010 accounted for 231 hospital episodes with ascites. The Norfloxacin group had 154 episodes and the Co-trimoxazole group had 77. The mean age of the cohort was 57.4 years (SD 12.4) and 62\% were male. Alcoholic cirrhosis was the major aetiology accounting for $79 \%$ of cases. The mean Child-Pugh and UKELD scores were 10.7 and 54 respectively. The overall incidence of SBP in our cohort was 19\%. Abstract P08 table 1 demonstrates that the incidence of hospital acquired SBP prophylaxis failure and mortality was not statistically different between the two therapies. However, selective bowel sterilisation with Co-trimoxazole did not lead to an increase $C$ difficle infection rate.

Abstract P08 Table 1 Outcome of patients hospitalised with cirrhosis and ascites

\begin{tabular}{llll}
\hline & Norfloxacin & Co-trimoxazole & p Value \\
\hline Episodes* & 134 & 67 & \\
Hospital acquired SBP & $9(6.71 \%)$ & $7(10.44 \%)$ & NS \\
Prophylaxis failure \% & $5 / 130(3.84 \%)$ & $4 / 64(6.24 \%)$ & NS \\
C difficle rate \% & $13(9.7 \%)$ & 0 & 0.009 \\
30-day mortality \% & $25(18.65 \%)$ & $15(22.38 \%)$ & NS \\
90-day mortality \% & $41(30.59 \%)$ & $16(23.88 \%)$ & NS \\
\hline
\end{tabular}

*Excluding patients with community acquired SBP

Conclusion Survival of cirrhotic patients with ascites is inversely related to severity of Liver disease, worsened with development of infections such as spontaneous bacterial peritonitis and $C$ difficle. This study shows that Co-trimoxazole inpatient prophylaxis against SBP is as effective as quinolone based regimes, but has the advantage of a dramatic reduction in $C$ difficle infection. At the same time the importance of measures like hand hygiene compliance, environmental cleanliness and strict policy of in hospital antibiotic prescribing cannot be underestimated.

\section{P09 VALIDATION OF A NOVEL BIOMARKER MODEL FOR THE PREDICTION OF NON-ALCOHOLIC STEATOHEPATITIS IN PATIENTS WITH NON-ALCOHOLIC FATTY LIVER DISEASE}

doi:10.1136/gutjnl-2011-300857a.9

${ }^{1} \mathrm{~S}$ Tanwar, ${ }^{2} \mathrm{P}$ Trembling, ${ }^{3}$ I N Guha, ${ }^{4} \mathrm{~J}$ Parkes, ${ }^{5} \mathrm{P}$ Kaye, ${ }^{6} \mathrm{~A} D$ Burt, ${ }^{7} \mathrm{~S} D$ Ryder, ${ }^{4} \mathrm{G}$ P Aithal, ${ }^{8} \mathrm{C}$ P Day, ${ }^{9} \mathrm{~W}$ M Rosenberg. ${ }^{7}$ Centre for Hepatology, University College, London, UK; ${ }^{2}$ Centre for Hepatology; ${ }^{3}$ University College London; ${ }^{4} U K ;{ }^{5}$ University Hospital; ${ }^{6}$ Queen's Medical Centre; ${ }^{7}$ Nottingham; ${ }^{8}$ Public Health Sciences and Medical Statistics; ${ }^{9}$ University of Southampton

Introduction The detection of non-alcoholic steatohepatitis (NASH) within non-alcoholic fatty liver disease (NAFLD) is important both 